\title{
Cuidados paliativos: inserção do ensino nas escolas médicas do Brasil
}

\author{
Inclusion of palliative care teaching in medical schools in Brazil
}

\author{
Andrea Augusta Castro ${ }^{1}$ (D) castro.andreaaugusta@gmail.com \\ Stella Regina Taquette ${ }^{1}$ (D) stella.taquette@gmail.com \\ Natan lório Marques ${ }^{1}$ (D) $\mid$ nataniorio19@gmail.com
}

\section{RESUMO}

Introdução: Abordagem em cuidados paliativos (CP) é uma modalidade assistencial recomendada pela Organização Mundial da Saúde. O sofrimento e o processo de morrer estão presentes no cotidiano da prática clínica, acometendo pessoas portadoras de doenças ameaçadoras à vida. Entretanto, o currículo predominante das escolas médicas brasileiras não inclui o ensino de CP.

Objetivos: Este estudo teve como objetivos conhecer os cursos de Medicina brasileiros que incluem CP em sua grade curricular e verificar de que forma estes vêm sendo ministrados.

Métodos: Trata-se de estudo descritivo e exploratório realizado por meio da busca de cursos de Medicina com disciplinas de CP nos sítios virtuais oficiais das instituições de ensino superior, no período de agosto a dezembro de 2018, e da análise das ementas disponíveis nas matrizes curriculares, no que diz respeito ao período oferecido, à carga horária, ao cenário e ao tipo de disciplina, se eletiva ou obrigatória.

Resultados: Das 315 escolas de Medicina cadastradas no Ministério da Educação, apenas 44 cursos de Medicina (14\%) dispõem de disciplina de CP. Esses cursos estão distribuídos em 11 estados brasileiros, 52\% estão na Região Sudeste, 25\% na Região Nordeste, 18\% na Região Sul, 5\% na Região Centro-Oeste, e nenhum na Região Norte. A modalidade predominante do tipo de disciplina foi obrigatória em $61 \%$ das escolas. Em relação à natureza, $57 \%$ são entidades privadas, percentual semelhante ao total de escolas médicas brasileiras. A disciplina ocorre no terceiro e quarto anos do curso, na maioria das instituições, e a carga horária mediana foi 46,9 horas. O cenário predominantemente é a sala de aula, e algumas instituições proporcionam a integração ensino-serviço-comunidade e prática médica. Os conteúdos programáticos são variados, incluindo tanatologia, geriatria, senescência e finitude, humanização, bioética, dor, oncologia e doenças crônicas.

Conclusão: $\mathrm{O}$ ensino de $\mathrm{CP}$ no Brasil é escasso, o que representa uma barreira à formação de médicos em consonância com as recomendações das entidades internacionais, das Diretrizes Curriculares Nacional e de marcos legais no âmbito do SUS. Fazem-se necessários investimentos das entidades médicas e dos organismos governamentais para a ampliação do ensino de CP e a consequente qualificação da formação médica.

Palavras-chave: Cuidados Paliativos; Educação Médica; Tanatologia; Currículo.

\section{ABSTRACT}

Introduction: The palliative care $(P C)$ approach is a care modality recommended by the World Health Organization. Suffering and the process of dying are present in everyday clinical practice, affecting people with life-threatening diseases. However, the predominant model of teaching in Brazilian medical schools does not include palliative care.

Objectives: The aim of the study was to get to know the Brazilian medical schools that include PC in their curriculum, and how it has been taught.

Methods: Descriptive and exploratory study, carried out by searching for medical schools with disciplines in PC, through the analysis of the course syllabi available in the curricular matrices on the official websites of higher education institutions from August to December 2018. They were analyzed considering the offered period of the PC content, workload, scenario, and type of discipline (elective or mandatory).

Results: 315 schools registered with the Ministry of Education were found, and only 44 of them (14\%) offer courses in PC. These schools are distributed throughout 11 Brazilian states, of which 52\% are located in the Southeast region, 25\% in the Northeast, $18 \%$ in the South, 5\% in the Midwest, and none in the North region. The predominant modality of the type of discipline in PC was mandatory in $61 \%$ of schools. Most Brazilian medical schools are private entities (57\%), a similar percentage to the total number of medical schools identified with the teaching of PC. This course takes place in the $3^{\text {rd }}$ and $4^{\text {th }}$ years of the course; in most schools, the workload was 46,9 hours. The predominant scenario is the classroom, while some institutions provide integration between teaching community service and medical practice. The program contents are diverse, including thanatology, geriatrics and finitude, humanization, bioethics, pain, oncology and chronic diseases.

Conclusion: PC education in Brazil is insufficient, which represents a barrier to the training of doctors in line with the recommendations of international entities, the National Curriculum Guidelines and legal frameworks within the scope of SUS. Investments by medical entities and government agencies are necessary to increase teaching in PC and the consequent qualification of medical training.

Keywords: Palliative Care; Medical Education; Thanatology; Curriculum.

${ }^{1}$ Universidade do Estado do Rio de Janeiro, Rio de Janeiro, Rio de Janeiro, Brasil.

Editora-chefe: Daniela Chiesa

Editor associado: Kristopherson Lustosa Augusto

Recebido em 19/08/20; Aceito em 17/02/21.

Avaliado pelo processo de double blind review. 


\section{INTRODUÇÃO}

Em razão do prolongamento da expectativa de vida e do consequente aumento da prevalência das doenças crônicodegenerativas causadas pelo envelhecimento da população, as sociedades atuais apresentam desafios no campo da saúde. Segundo dados da Organização Mundial da Saúde (OMS), essa realidade traz à tona a importância da inclusão na formação médica de temas relacionados aos cuidados de fim de vida e ao processo de morrer. As doenças emergentes, a exemplo da coronavirus disease 2019 (Covid-19), aliadas às transições demográficas e epidemiológicas, exigem processos de cuidado específicos durante o adoecimento e a morte. Pessoas em finitude, independentemente de estarem em processos agudos ou serem portadoras de doenças avançadas, podem se beneficiar da abordagem em cuidados paliativos (CP), o que é corroborado por evidências científicas. $\mathrm{O}$ apoio adequado de profissionais com os recursos dos CP exige o repensar do papel das escolas médicas visando atender às necessidades de saúde dos pacientes e da família deles ${ }^{1-4}$.

A abordagem em CP emerge de um processo histórico e sociocultural, tendo os hospices medievais, locais de hospedagem destinados a atender pessoas que necessitavam de cuidados por enfermidade ou fome, como exemplos dessa modalidade. Contudo, somente nos anos 1960, nascem os CP modernos. Duas profissionais de saúde, Cicely Saunders, no Reino Unido, e Elizabeth Kübler-Ross, na Suíça e nos Estados Unidos, impulsionaram o ensino e pesquisa em CP visando às melhores práticas para pessoas que necessitavam de uma assistência na finitude da vida ${ }^{5,6}$.

No Brasil, registra-se o pioneirismo do professor Marco Túlio A. Figueiredo, em curso eletivo para alunos em CP na Universidade Federal de São Paulo, de 1994 a 2008. Segundo Oliveira et al7, em avaliação do ensino em bioética e $C P$, constata-se um frágil compromisso das escolas médicas brasileiras em relação ao ensino de CP.

A abordagem em $\mathrm{CP}$ pode ser compreendida como uma modalidade assistencial, reconhecida como adequada para atuar em crianças e adultos em situação de sofrimento e portadores de doenças ameaçadoras à vida e também aos seus familiares. Os CP devem ser oferecidos às pessoas em condições que levem a alto risco de mortalidade, impacto negativo na qualidade de vida e repercussões na funcionalidade do corpo. Os CP levam em consideração a pessoa de forma integral, nos aspectos físicos, psíquicos, sociais e espirituais, e não apenas sua doença. Para serem efetivados, requerem trabalho interprofissional e interdisciplinar para o manejo de sintomas e a prevenção de complicações ${ }^{8,9}$.

A intensificação da capacitação em CP é pertinente no ensino desde a graduação, sendo uma estratégia que corrobora a desospitalização e maior satisfação dos usuários. O ensino de $\mathrm{CP}$, quando está incluído na graduação, desperta a atenção dos futuros médicos, melhorando o cuidado oferecido ao paciente. $\mathrm{Na}$ interação com pacientes e familiares, observam-se maior senso de controle, compaixão, empatia e respeito por meio da exposição aos pacientes com doenças avançadas ${ }^{10,11}$.

Observa-se que as apresentações dos currículos são muito variadas no mundo, mesmo em países onde a medicina paliativa tornou-se obrigatória nas escolas médicas na graduação. SegundoVon Gunten et al ${ }^{12}$ eSchulzet al ${ }^{13}$, mudanças nas atitudes são importantes desafios, requerendo alternância de estratégias didáticas e vivenciais, potencializadas pela autorreflexão e pelo ensino interdisciplinar para serem alcançadas as competências exigidas no processo de aprendizagem em CP.

As competências em CP estão alinhadas com a atenção centrada na pessoa, o respeito à autonomia e a abordagem relacionada à família. Essas competências envolvem questões técnicas, culturais e éticas, tais como a diminuição de uso inapropriado e pouco efetivo de intervenção médica invasiva em detrimento de recursos para melhorar a qualidade de vida e o lidar com o processo de morte na existência humana ${ }^{14,15}$.

Conforme a literatura internacional, a avaliação do ensino de CP demonstra mudanças de valores e atitudes nos estudantes que são reconhecidas como essenciais para a formação médica, contribuindo para a aquisição de habilidades de comunicação, a exploração das preferências do doente para o cuidado no fim de vida, a transmissão de más notícias e a conversação sobre questões espirituais e suas práticas ${ }^{16-18}$.

De acordo com MacPherson et $\mathrm{al}^{19}$, o ensino de CP deve estar integrado no currículo da graduação, possibilitando melhor manejo de sintomas, trabalho em equipe e cuidado com foco na pessoa desde as etapas iniciais do adoecimento.

Segundo Toledo et $\mathrm{al}^{20}$, existem poucos dados sobre o ensino de CP no Brasil, e uma literatura escassa corrobora esse panorama. Os autores tecem comentários sobre a identificação de barreiras no ensino de CP e apontam os desafios enfrentados pelos coordenadores dos cursos de Medicina, como falta de corpo docente especializado, ausência de serviço clínico de $C P$, reduzido interesse da instituição, verbas insignificantes e escassez de tempo e de material didático apropriado.

Diante dos dados apresentados, o ensino de CP se mostra uma necessidade para a formação de médicos qualificados. Esse quadro desperta os seguintes questionamentos:

- Como está o ensino de CP nas escolas brasileiras?

- Quais projetos pedagógicos impulsionam a formação dos profissionais de saúde voltados para cuidados, transformando o foco da atenção da cura para o cuidado? 
O presente estudo objetiva conhecer os cursos de Medicina brasileiros que incluem os CP em sua grade curricular e verificar de que forma estes vêm sendo ministrados.

\section{MÉTODOS}

O presente estudo é descritivo, exploratório e transversal, com abordagem quantitativa por meio de dados secundários sobre as escolas de ensino médico com inserção no ensino de $\mathrm{CP}$ no Brasil. O universo da pesquisa concentra-se nas escolas médicas que possuem na grade curricular ensino de CP. Iniciou-se pela busca de todas as escolas médicas cadastradas no banco de dados oficiais brasileiros disponíveis no sistema eletrônico do Ministério da Educação do Brasil (Cadastro e-MEC de Instituições e Cursos de Educação Superior) e nos sites das escolas médicas disponíveis (www.escolasmedicas.com.br)21,22. Em seguida, foram visitados os sites de cada escola para análise e seleção das que possuem disciplina de CP na matriz curricular, no período de agosto a dezembro de 2018. A fim de identificar quais escolas dispõem de disciplina de CP em suas matrizes curriculares, utilizaram-se na busca as seguintes temáticas: cuidados paliativos, tanatologia, processos de morte, finitude e morte, oncologia e $C P$, geriatria e $C P$, envelhecimento e morte.

Quanto ao perfil de distribuição das escolas, analisaramse os seguintes aspectos: região administrativa e unidades da Federação (UF) - número de vagas ofertadas no primeiro ano -; natureza da instituição, se pública ou privada; e, entre as públicas, qual o tipo de administração, se federal, estadual ou municipal. A mesma análise de perfil foi feita em relação aos cursos de Medicina que dispõem de disciplina de CP em sua matriz curricular. Para análise das ementas das disciplinas oferecidas, utilizamos as seguintes variáveis: período oferecido, carga horária, cenário de prática e tipo de disciplina (eletiva ou obrigatória), tendo como base as recomendações de entidades internacionais para o ensino de CP e as Diretrizes Curriculares Nacionais (DCN) para os Cursos de Medicina de 201423,24.

\section{RESULTADOS}

Foi identificada a existência de 315 escolas de Medicina em funcionamento no Brasil, no período estudado. Quanto à distribuição geográfica, 42,5\% estão localizadas na Região Sudeste, $16,5 \%$ na Região Sul, $8,9 \%$ na Região Centro-Oeste, $23,9 \%$ na Região Nordeste e 8,2 \% na Região Norte. As escolas estão distribuídas em todos os estados brasileiros, sendo a maioria dos cursos privados (63,8\%). Foram disponibilizadas aproximadamente 33.520 vagas de primeiro ano nos cursos de Medicina em 2018, sendo quase metade (46\%) na Região Sudeste, como pode ser visualizado na Tabela 1.

Os cursos de Medicina de natureza pública foram 114 (36,2\%). No Amapá e em Roraima, 100\% das escolas médicas são públicas. A maioria dos cursos de Medicina são de gestão privada (201), dos quais 105 (52\%) encontram-se na Região Sudeste.

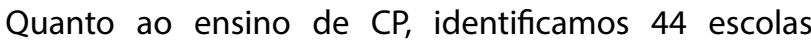
médicas que dispõem de disciplina de $C P$ em suas matrizes curriculares apresentadas nos sites oficiais das instituições de ensino, o que corresponde a $14 \%$ do total de escolas e $16 \%$ do total de vagas ofertadas de primeiro ano no curso de Medicina.

Tabela 1. Distribuição das escolas médicas segundo distribuição geográfica (região-UF), número de vagas no primeiro ano e natureza da instituição - Brasil, 2018.

\begin{tabular}{|c|c|c|c|c|c|c|}
\hline \multirow{2}{*}{ Região } & \multirow{2}{*}{ UF } & \multirow{2}{*}{$\begin{array}{c}\text { No } \\
\text { vagas }\end{array}$} & \multicolumn{2}{|c|}{$\begin{array}{l}\text { Escolas } \\
\text { públicas }\end{array}$} & \multicolumn{2}{|c|}{$\begin{array}{c}\text { Escolas } \\
\text { particulares }\end{array}$} \\
\hline & & & $\mathrm{n}$ & $\%$ & $\mathrm{n}$ & $\%$ \\
\hline \multirow{8}{*}{ NORTE } & PA & 690 & 4 & 67 & 2 & 33 \\
\hline & $\mathrm{AM}$ & 465 & 3 & 60 & 2 & 40 \\
\hline & RO & 325 & 1 & 25 & 3 & 75 \\
\hline & $A C$ & 161 & 1 & 50 & 1 & 50 \\
\hline & AP & 60 & 1 & 100 & 0 & 0 \\
\hline & TO & 518 & 2 & 33 & 4 & 67 \\
\hline & $\mathrm{RR}$ & 140 & 2 & 100 & 0 & 0 \\
\hline & Total & 2.359 & 14 & 60 & 12 & 40 \\
\hline \multirow{10}{*}{ NORDESTE } & $\mathrm{BA}$ & 2.164 & 11 & 52 & 10 & 48 \\
\hline & $P E$ & 1.490 & 6 & 55 & 5 & 45 \\
\hline & PB & 985 & 3 & 33 & 6 & 67 \\
\hline & CE & 1.093 & 4 & 50 & 4 & 50 \\
\hline & $\mathrm{PI}$ & 631 & 5 & 71 & 2 & 39 \\
\hline & RN & 472 & 2 & 40 & 3 & 60 \\
\hline & MA & 539 & 2 & 33 & 4 & 67 \\
\hline & $\mathrm{AL}$ & 495 & 2 & 40 & 3 & 60 \\
\hline & SE & 320 & 2 & 67 & 1 & 33 \\
\hline & Total & 8.189 & 37 & 49 & 38 & 51 \\
\hline \multirow{5}{*}{ CENTRO-OESTE } & GO & 1.374 & 4 & 31 & 9 & 69 \\
\hline & DF & 336 & 2 & 50 & 2 & 50 \\
\hline & MT & 431 & 4 & 67 & 2 & 33 \\
\hline & MS & 388 & 4 & 80 & 1 & 20 \\
\hline & Total & 2.529 & 14 & 47 & 14 & 53 \\
\hline \multirow{5}{*}{ SUDESTE } & SP & 7.300 & 9 & 15 & 51 & 85 \\
\hline & MG & 4.787 & 14 & 30 & 32 & 70 \\
\hline & RJ & 2.789 & 5 & 23 & 17 & 77 \\
\hline & ES & 718 & 1 & 17 & 5 & 83 \\
\hline & Total & 15.594 & 29 & 22 & 105 & 78 \\
\hline \multirow{4}{*}{ SUL } & PR & 2.139 & 9 & 45 & 11 & 55 \\
\hline & SC & 957 & 4 & 33 & 8 & 67 \\
\hline & RS & 1.753 & 7 & 35 & 13 & 65 \\
\hline & Total & 4.849 & 20 & 38 & 32 & 62 \\
\hline Total geral & & 33.520 & 114 & 36 & 201 & 64 \\
\hline
\end{tabular}


A distribuição dessas escolas segundo região, UF, número de vagas oferecidas no primeiro ano e natureza da instituição pode ser visualizada na Tabela 2. Vale destacar que, na Região Norte, nenhum curso apresenta ensino de $C P$, diferentemente da Região Sudeste, na qual se concentra a maioria de escolas com essa disciplina (52\%).

Dentre as 44 instituições que dispõem de ensino de CP

Tabela 2. Distribuição das escolas médicas com ensino de CP segundo distribuição geográfica (região-UF), número de vagas no primeiro ano e natureza da instituição Brasil, 2018.

\begin{tabular}{|c|c|c|c|c|c|c|}
\hline \multirow{2}{*}{ Região } & \multirow{2}{*}{ UF } & \multirow{2}{*}{$\begin{array}{c}\text { No } \\
\text { vagas }\end{array}$} & \multicolumn{2}{|c|}{$\begin{array}{l}\text { Escolas } \\
\text { públicas }\end{array}$} & \multicolumn{2}{|c|}{$\begin{array}{c}\text { Escolas } \\
\text { particulares }\end{array}$} \\
\hline & & & $\mathbf{n}$ & $\%$ & $\mathbf{n}$ & $\%$ \\
\hline \multirow{4}{*}{ NORDESTE } & BA & 525 & 3 & 50 & 3 & 50 \\
\hline & CE & 460 & 2 & 50 & 2 & 50 \\
\hline & $\mathrm{RN}$ & 80 & 1 & 100 & 0 & 0 \\
\hline & Total & 1.065 & 6 & 55 & 5 & 45 \\
\hline \multirow{2}{*}{ CENTRO-OESTE } & MS & 140 & 2 & 100 & 0 & 0 \\
\hline & Total & 140 & 2 & 100 & 0 & 100 \\
\hline \multirow{5}{*}{ SUDESTE } & SP & 986 & 4 & 67 & 2 & 33 \\
\hline & MG & 945 & 1 & 17 & 5 & 83 \\
\hline & RJ & 1.309 & 2 & 22 & 7 & 78 \\
\hline & ES & 228 & 2 & 50 & 2 & 50 \\
\hline & Total & 3.468 & 7 & 28 & 16 & 64 \\
\hline \multirow{4}{*}{ SUL } & PR & 620 & 3 & 50 & 3 & 50 \\
\hline & SC & 100 & 1 & 100 & 0 & 0 \\
\hline & RS & 100 & 0 & 0 & 1 & 100 \\
\hline & Total & 820 & 4 & 50 & 4 & 50 \\
\hline Total geral & & 5.493 & 19 & 43 & 25 & 57 \\
\hline
\end{tabular}

na matriz curricular, a disciplina é obrigatória em 27 (61\%) e eletiva em 17 (39\%).

Na maioria das escolas, o ensino de CP ocorre no ciclo clínico (terceiro e quarto anos), com uma mediana na carga horária de 46,9 horas, e observou-se uma variação da carga horária entre 18 e 119 horas (Gráfico 1). Na estimativa, foram excluídas as escolas que apresentam caráter modular. $O$ cenário de ensino mais frequente é a sala de aula, ou seja, predominantemente teórico. Observamos diversidade de cenário em sete instituições, com integração ensino-serviço- comunidade e prática médica. O ensino de CP está inserido no formato de módulos (carga horária > 120 horas) em 12 escolas, compondo teoria e prática, não podendo ser identificada carga horária específica em CP. Em duas escolas, o ensino de CP é apresentado em mais de um momento do curso no formato de disciplina. Em duas escolas, não foram disponibilizadas as ementas, apenas constam na matriz curricular. Conforme apontam as ementas das escolas estudadas, os conteúdos predominantes foram tanatologia, geriatria, senescência e finitude, humanização, ética-bioética, dor, oncologia e doenças crônicas.

\section{DISCUSSÃO}

O estudo demonstra que a distribuição geográfica das escolas de Medicina que dispõem de ensino de CP é semelhante à do total de escolas no país, com exceção da Região Norte, onde não foi observado nenhum curso com disciplina de CP. A maior concentração de cursos de Medicina ocorre na Região Sudeste, o que pode ser um indicador das iniquidades no país (Tabela 2).

Quando se observa a distribuição das escolas com ensino de CP no país, constata-se maior investimento em alguns estados. No Ceará e no Rio de Janeiro, 50\% e 41\% das escolas dispõem, respectivamente, de disciplinas de CP. Em Mato Grosso do Sul, observa-se o ensino de CP em 40\% das escolas médicas.

Gráfico 1.Distribuição da carga horária das disciplinas de CP nas escolas médicas do Brasil - 2018.

Carga horária por disciplina $\quad$ Carga horária média

125

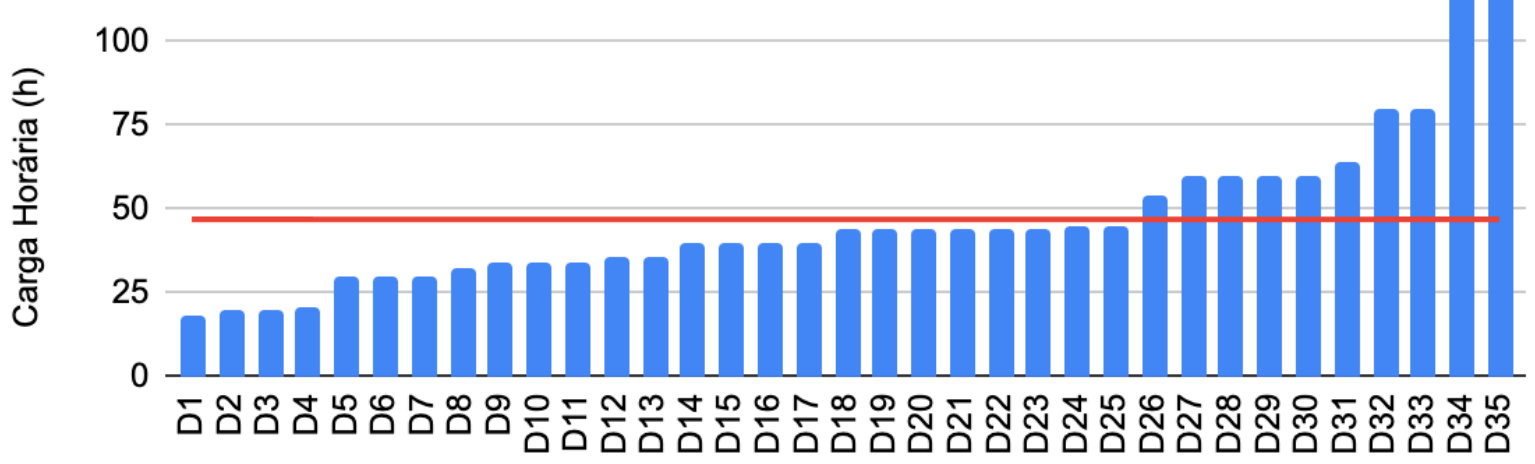

Disciplina (D) de cuidados paliativos 
Por sua vez, São Paulo conta com 10\% de escolas com disciplina de CP. Evidenciam-se diferenças inter e intrarregionais. Na Região Sul, 30\% das escolas do Paraná apresentam disciplina de $\mathrm{CP}$, e, no Rio Grande do Sul, há somente $5 \%$. Essa realidade pode ser reflexo de marcos legais de fomento aos $\mathrm{CP}$, tanto no âmbito nacional como no estadual. Iniciativas da sociedade e do Poder Legislativo estadual, a exemplo do que acontece no Paraná e Rio de Janeiro, reforçam o fomento de políticas públicas para a reorganização dos serviços e formação de recursos humanos no campo dos $\mathrm{CP}^{25-27}$.

Em relação às escolas médicas com disciplina de $C P$, observa-se que 25 (57\%) são privadas. Essa proporção em relação à entidade mantenedora também é observada no total de escolas médicas brasileiras, o que traduz uma tendência de progressão pela privatização no ensino médico, conforme descrito na literatura ${ }^{28}$.

Os estudos internacionais apontam consenso mundial sobre a importância do ensino de CP na graduação. Contudo, sua inserção e consolidação do processo do ensinoaprendizagem são muito diversas em cada país e escola. As realidades são muito distintas em face dos aspectos culturais, econômicos, políticos e sociais. Embora seja considerado importante, existem desafios para a inserção do ensino de CP em todo o mundo. Na América Latina, somente cinco países têm certificação oficial em CP. No Brasil, a medicina paliativa é considerada uma área de atuação29,30. Nos Estados Unidos, o ensino específico de CP é declarado em três quartos das escolas médicas, percentual bem superior ao encontrado no Brasil, onde identificamos essa disciplina em $14 \%$ das escolas.

Floriani aponta a importância de uma política de ensino com programas educacionais continuados em CP, em consonância com outras experiências mundiais. A inclusão de disciplina de CP pode diminuir as distorções ante as limitações terapêuticas curativas, reforçando a relação do cuidado e outras abordagens não curativas de doenças em fase avançada ${ }^{31,32}$.

De acordo com Calda et al33, os desafios da implementação do ensino de CP podem ser atribuídos ao desconhecimento sobre $\mathrm{CP}$, gerando resistência a mudanças, o que é persistente pela baixa capacitação do corpo docente nessa área de conhecimento. Por sua vez, os currículos possuem carga horária excessiva e recursos limitados.

Os conteúdos programáticos encontrados nas ementas das escolas brasileiras apontam para os domínios preconizados pelas entidades científicas e pelos estudos nacionais e internacionais. Algumas escolas enfatizam a tanatologia; outras abordam o ciclo de vida, como a geriatria, senescência e finitude; e outras tratam de grupos de doenças mais específicas, como a oncologia, e de manejo de sintomas, como a dor. Segundo MacPherson et $\mathrm{al}^{34}$, o ensino deve estar integrado no currículo da graduação, e recomenda-se a inclusão de áreas de conhecimentos como controle de sintomas, trabalho em equipe e cuidado da pessoa desde as etapas básicas do adoecimento.

As experiências brasileiras de ensino de CP apresentam uma mediana de carga horária de 46,9 horas, em consonância com o preconizado, embora com variações nos formatos modulares. Conforme entidades internacionais, como a Worldwide Palliative Care Alliance (WPCA), e recomendações da Academia Nacional de Cuidados Paliativos, a capacitação dos profissionais, pode ser dividida em três níveis, sendo preconizada a formação básica em CP na graduação de todas a áreas da saúde, podendo variar de 18 a 45 horas a carga horária específica ${ }^{35,36}$.

A literatura recomenda o aprendizado baseado em experiências que geram prática significativa. Segundo Shaheen et $\mathrm{al}^{37}$, nos Estados Unidos os temas trabalhados são ministrados por médicos de diversas especialidades, como geriatras, médicos de família na atenção domiciliar e clínicos nas enfermarias. Contudo, são relatadas dificuldades em estratégias de ensino, ainda predominando táticas de leitura e discussão em pequenos grupos.

Em relação aos cenários de aprendizado, foi observada, em algumas experiências brasileiras, a integração ensinoserviço-comunidade e prática médica. Considerando os estudos de Costa et $\mathrm{al}^{38}$, as transformações da representatividade dos estudantes sobre $\mathrm{CP}$, o tempo requerido para a racionalização $\mathrm{e}$ sensibilização, e a aquisição das competências assertivas como manejo de sintomas são potencializados pela reflexão teórica associada ao campo de prática e ao ensino interprofissional.

Esse conhecimento pode ser empregado em todas as áreas de atuação e especialidades dos futuros médicos. Para Gibbins et $\mathrm{al}^{39}$, a inserção do ensino de CP na graduação possibilita ao estudante desenvolver competências que irão melhorar o cuidado do paciente não só na finitude, mas também na assistência geral destinada a ele.

Segundo Carroll et $\mathrm{al}^{40}$, há falta de padronização quanto aos objetivos de aprendizagem e ao que deve ser ministrado sobre $\mathrm{CP}$, assim como a fragmentação do ensino e/ ou sua ausência formal nos currículos. Diferentes estratégias de aprendizagem podem ser inseridas durante a formação dos médicos em CP gerais, e não apenas como uma especialidade.

Embora seja ainda escasso o ensino de CP no Brasil, dados apontam que estão ocorrendo transformações no país. Até 2012, o país registrava oficialmente três escolas (2\%) com disciplina de CP em sua grade curricular. Os estudos de Oliveira et $\mathrm{al}^{7}$ e Fonseca et $\mathrm{al}^{41}$, evidenciaram iniciativas pontuais, embora exitosas, no campo dos CP em algumas escolas de Minas Gerais, São Paulo e Rio Grande do Sul. A ocorrência do ensino sistematizado em CP em 11 UF e na maioria das regiões 
do Brasil evidencia expansão do ensino de CP nas escolas brasileiras. Segundo levantamento realizado pela Academia Nacional de Cuidados Paliativos em 2017, observa-se um crescimento de 40 equipes em CP para 123 serviços em CP nos últimos 15 anos, e novos estudos estão em andamento para atualização ${ }^{42}$.

\section{CONCLUSÃO}

Verificamos neste estudo a expansão do ensino de CP no Brasil, porém ainda de forma insuficiente, e uma maior preocupação para a inserção de eixo humanista, visando à aquisição de competências centrais na abordagem em CP. Ressaltamos a necessidade de desdobramentos desta pesquisa em futuras investigações para avaliar as experiências exitosas na área e o papel dos diferentes atores sociais, de modo a ampliar o conhecimento do protagonismo estudantil, as estratégias dos docentes envolvidos e a compreensão dos gestores quanto à potencialidade transformadora do ensino de CP.

Apesar de limitado às informações contidas nas ementas das matrizes curriculares das escolas de Medicina que são heterogêneas e por vezes reduzidas, nosso estudo revela que a escassez do ensino de CP representa uma barreira à formação de médicos em consonância com as recomendações das entidades internacionais, das DCN e de marcos legais no âmbito do Sistema Único de Saúde (SUS). Fica evidenciada a urgência de investimentos das entidades médicas e dos organismos governamentais para a ampliação do ensino de CP e consequente qualificação da formação médica.

\section{CONTRIBUIÇÃO DOS AUTORES}

Andrea Augusta Castro e Stella Regina Taquette participaram igualmente na concepção e no desenho do trabalho, e na aquisição, análise e interpretação dos resultados da pesquisa. Natan lório Marques participou na aquisição e organização dos dados.

\section{CONFLITO DE INTERESSES}

Os autores declaram não haver conflito de interesses neste estudo.

\section{FINANCIAMENTO}

O estudo foi financiado pela Fundação de Amparo à Pesquisa do Estado do Rio de Janeiro (Faperj) e submetido ao "Edital Faperj 12/2018 - Programa Apoio às Universidades Estaduais UERJ, UENF e ENZO".

\section{REFERENCES}

1. Helman CG. Cultura, saúde \& doença. 4a ed. Porto Alegre: Artmed; 2003.

2. FAIMER. Consenso global das responsabilidades sociais das escolas médicas. 2012 [access in 3 nov 2017]. Available from: http://healthsocialaccountability.sites.olt.ubc.ca/files/2012/02/GCSA-Global-Consensus-document_portuguese.pdf.
3. Kelley AS. Defining serious illness. J Palliat Med. 2014;17(9):985. doi: 10.1089/jpm.2014.0164.

4. Gomes ALZ, Othero MB. Cuidados paliativos. Estud Av. 2016;30(88):15566. doi: 10.1590/S0103-40142016.30880011.

5. Twycross R. Medicina paliativa: filosofia e considerações éticas. Acta Bioeth. 2000;6(1):27:46. doi: 10.1590/S0103-40142016.30880011.

6. Floriani CA. Moderno movimento hospice: kalotanásia e o revivalismo estético da boa morte. Rev Bioét. 2013;21(3):397-404. doi: 10.1590/S1983 80422013000300003.

7. de Oliveira JR, Ferreira AC, de Rezende NA. Ensino de bioética e cuidados paliativos nas escolas médicas do Brasil. Rev Bras Educ Med. 2013;37(2):285-90 [access in 20 mar 2018]. Available from: https://www. scielo.br/pdf/rbem/v37n2/17.pdf.

8. Burlá C, Py L. Cuidados paliativos: ciência e proteção ao fim da vida. Cad Saude Publica. 2014; 30(6):1-3. doi: 10.1590/0102-311XPE020614.

9. Bifulco VA, Lochida LC. A formação na graduação dos profissionais de saúde e a educação para cuidado de pacientes fora de recursos terapêuticos de cura. Rev Bras Educ Med. 2009;33(1):92-100 [access in 12 apr 2019]. Disponívelem:https://www.scielo.br/scielo.php?pid=S01005502200900010 0013\&script=sci_abstract\&tlng=pt.

10. Crawford GB, Zambrano SC. Junior doctors views of how their un dergraduate clinical electives in palliative care influenced their current practice of medicine. Acad Med. 2015;90(3):338-44. doi: 10.1097/ ACM.0000000000000632.

11. Costa AP, Poles K, Silva AE. Palliative care education: experience of medical and nursing students. Interface (Botucatu). 2016;20(59):1041-52. doi: 10.1590/1807-57622015.0774.

12. Von Gunten CF, Mullan P, Nelesen RA, Soskins M, Savoia M, Buckholz G, et al. Development and evaluation of a palliative medicine curriculum for third-year medical students. J Palliat Med. 2012; 15(11):1198-217. doi: 10.1089/jpm.2010.0502.

13. Schulz C, Möller MF, Seidler D, Schnell MW. Evaluating an evidence-based curriculum in undergraduate palliative care education: piloting a phase II exploratory trial for a complex intervention. BMC Med Educ. 2013;(4)13:1. doi:10.1186/1472-6920-13-1.

14. Silva MLSR. O papel do profissional da atenção primária à saúde em cuidados paliativos. Rev Bras Med Fam Comunidade. 2014;9(30): 45-53. doi: $10.5712 /$ rbmfc9(30)718.

15. Gibbins J, McCoubrie R, Maher J, Forbes K. Incorporating palliative care into undergraduate curricula: lessons for curriculum development. Med Educ. 2009;43(8):776-83. doi: 10.1111/j.1365-2923.2009.03400.x.

16. Parikh PP, White MT, Buckingham L, Tchorz KM. Evaluation of palliative care training and skills retention by medical students. J Surg Res. 2017;211:1727. doi: 10.1016/j.jss.2016.11.006.

17. Simmenroth-Nayda A, Alt-Epping B, Gágyor I. Breaking bad news - an interdisciplinary curricular teaching-concept. GMS Z Med Ausbild 2011;28(4):doc 52. doi: 10.3205/zma000764.

18. Sweeney C, Lynch G, Khashan A, Maher B, Murphy M, O'Brien T. The impact of a medical undergraduate student-selected module in palliative care. BMJ Support Palliat Care. 2014;4(1):92-7. doi: 10.1136/bmjspcare-2012-000283.

19. MacPherson A, Lawrie I, Collins S, Forman L. Teaching the difficult-to-teach topics. BMJ Support Palliat Care. 2014;4(1):87-91. doi: 10.1136/bmjspcare-2012-000408.

20. Toledo AP, Priolli DG. Cuidados no fim da vida: o ensino médico no Brasil. Rev Bras Educ Med. 2012;36(1):109-17 [access in 13 sep 2020]. Available from: https://www.scielo.br/scielo.php?pid=S0100-55022012000100015 \&script=sci_abstract\&tlng=pt.

21. Brasil. Sistema e-MEC. Instituições de Educação Superior e Cursos Cadastrados. Brasília: MEC; 2017 [access in 17 dec 2017]. Available from http:// emec.mec.gov.br/.

22. Nassif ACN. Escolas médicas do Brasil. São Paulo: Nassif; 2018 [access in 19 aug 2018]. Available from: http://www.escolasmedicas.com.br/estat.php. 
23. Knaul FM, Farmer PE, Krakauer EL, De Lima L, Bhadelia A, Kwet JX, et al. Alleviating the access abyss in palliative care and pain relief-an imperative of universal health coverage: the Lancet Commission report. Lancet. 2017(7);391(10128):1391-444. doi: 10.1016/S0140-6736(17)32513-8.

24. Brasil. Resolução no 3, de 20 de junho de 2014. Institui Diretrizes Curriculares Nacionais do Curso de Graduação em Medicina e dá outras providências. Brasília: Ministério da Educação; 2014 [access in 16 apr 2020]. Available from: http://portal.mec.gov.br/.

25. Brasil. Resolução $n^{\circ} 41$, de 31 outubro de 2018. Dispõe sobre as diretrizes para a organização dos cuidados paliativos, à luz dos cuidados continuados integrados, no âmbito do Sistema Único de Saúde. Diário Oficial da União; 23 nov 2018. Seção 1, p. 276 [access in 17 mar 2020]. Available from: https://www.jusbrasil.com.br/diarios/218740387/dou-secao-1-2311-2018-pg-276.

26. Rio de Janeiro. Lei no 9449 , de 5 de julho de 2019. Dispõe sobre a criação do Programa Estadual de Cuidados Paliativos no âmbito do Estado do Rio de Janeiro. DOE; 8 jul 2019 [access in 17 mar 2020]. Available from: http:// alerjln1.alerj.rj.gov.br/scpro1519.nsf.

27. Assembleia Legislativa do Estado do Paraná. Lei no 20.091, de 19 de dezembro de 2019. Dispõe sobre a garantia de equipe multidisciplinar em cuidados paliativos. Diário Oficial; 19 dez 2019 [access in 20 mar 2020]. Available from: https://www.assembleia.pr.leg.br/comunicacao/noticias/ lei-garante-cuidados-paliativos-a-pacientes-com-doencas-graves-no-parana.

28. Scheffer M, Cassenote A, Guilloux AGA, Biancarelli A, Miotto BA, Mainardi G, et al. Demografia médica no Brasil 2018. São Paulo: Departamento de Medicina Preventiva da Faculdade de Medicina da USP, Conselho Regional de Medicina do Estado de São Paulo, Conselho Federal de Medicina; 2018 [access in 17 mar 2020). Available from: https://jornal.usp.br/wp-content/ uploads/DemografiaMedica2018.pdf.

29. Pastrana T, De Lima L, Wenk R, Eisenchlas J, Monti C, Rocaforte J, et al. Atlas de Cuidados Paliativos na América Latina. Houston: IAPC; 2012. [access in 10 mar 2020]. Available from: https://cuidadospaliativos.org/uploads/2014/1/Atlas\%20Portugues.pdf.

30. Conselho federal de Medicina. Resolução CFM no 1.973/2011. Dispõe sobre a nova redação do Anexo II da Resolução CFM n 1.845/08, que celebra o convênio de reconhecimento de especialidades médicas firmado entre o Conselho Federal de Medicina (CFM), a Associação Médica Brasileira (AMB) e a Comissão Nacional de Residência Médica (CNRM). Diário Oficial da União; $1^{\circ}$ ago 2011. Seção I, p. 144-7 [access in 13 mar 2020]. Available from: http://www.cremesp.org.br/?siteAcao=PesquisaLegislacao\&dif $=$ s\&ficha $=1 \&$ id $=9791 \&$ tipo $=$ RESOLU\%C7\%C3O\&orgao $=$ Consel ho\%20Federal\%20de\%20Medicina\&numero=1960\&situacao=VIGENTE\&data $=16-12-2010 \&$ vide $=$ sim.
31. Floriani CA. Palliative care in Brazil: a challenge to the health care system. Palliat Care. 2008;2: 19-24. doi: 10.1177\%2F117822420800200001.

32. Floriani CA, Schramm FR. Desafios morais e operacionais da inclusão dos cuidados paliativos na rede de atenção básica. Cad Saude Publica. 2007;23(9):2072-80. doi: 10.13140/2.1.3201.5367.

33. Caldas GHO, Moreira SNT, Vilar MJ Cuidados paliativos: uma proposta para o ensino da graduação em Medicina. Rev Bras Geriatr Gerontol. 2018;21(3):269-80. doi: 10.1590/1981-22562018021.180008.

34. MacPherson A, Lawrie L, Collins S, Forman L. Teaching the difficult-to-teach topics. BMJ Support Palliat Care. 2014;4(1):87-91. doi: 10.1136/bmjspcare-2012-000408.

35. World Health Organization. Global Atlas of Palliative Care at the end-of-life. Geneva: WHO, 2014. [access in 2 dec 2014]. Available from: http://www. who.int/nmh/Global_Atlas_of_Palliative_Care.pdf.

36. Lehto JT, Hakkarainen K, Kellokumpu-Lehtinen PL, Saarto T. Undergraduate curriculum in palliative medicine at Tampere University increases students' knowledge. BMC Palliat Care. 2017; 16(1): 9-13 doi: 10.1186/s12904-016-0182-8.

37. Shaheen AW, Denton GD, Stratton TD, Hoellein AR, Chretien KC. End-of-life and palliative care curricula in internal medicine clerkships: a report on the presence, value, and design of curricula as rated by clerkship directors. Acad Med. 2014; 89(8):1168-73. doi: 10.1097/ACM.00000000000000311.

38. Costa $A P$, Poles $K$, Silva AE. Formação em cuidados paliativos: experiência de estudantes de medicina e enfermagem. Interface (Botucatu). 2016;20(59):1041-52. doi: 10.1590/1807-57622015.0774.

39. Gibbins J, McCoubrie R, Maher J, Wee B, Forbes K. Recognizing that it is part and parcel of what they do: teaching palliative care to medical students in the UK. Palliat Med. 2010;24(3):299-30. doi: 10.1177/0269216309356029.

40. Carroll T, El-Sourady M, Karlekar M, Richeson A. Primary palliative care education programs: review and characterization. Am J Hosp Palliat Care. 2019 June;36(6):546-49. doi: 10.1177/1049909118809947.

41. Fonseca A, Geovanini F. Cuidados paliativos na formação do profissional da área de saúde. Rev Bras Educ Med. 2013;37(1):120-5. doi: 10.1590/ S0100-55022013000100017.

42. Mendes EC. Cuidados paliativos e câncer: uma questão de direitos humanos, saúde e cidadania [dissertação]. Rio de Janeiro. Fundação Oswaldo Cruz, Escola Nacional de Saúde Pública Sergio Arouca, 2017 [access in 12 oct 2020]. Available from: https://www.arca.fiocruz.br/bitstream/ icict/24828/2/ernani_costa.pdf. 